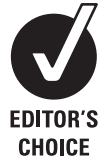

${ }^{1}$ Retina Center, Department of Ophthalmology, New York Eye and Ear Infirmary, New York, USA; ${ }^{2}$ Department of Ophthalmology, Kaplan Medical Center, Rehovot, Israel;

${ }^{3}$ Department of Ophthalmology, New York Medical College,

Valhalla, New York, USA

Correspondence to: Dr R B Rosen, Department of Ophthalmology, New York Eye and Ear Infirmary, 310 East 14th Street, New York City, NY 10003, USA; rrosen@nyee.edu

Accepted 12 July 2009 Published Online First 18 August 2009

\title{
New patterns of retinal collateral circulation are exposed by a retinal functional imager (RFI)
}

\author{
G Landa, ${ }^{1,2,3}$ R B Rosen ${ }^{1,3}$
}

\section{ABSTRACT}

Aims: To describe different patterns of retinal collateral circulation observed in normal subjects and in patients with a variety of ocular diseases during studies with the retinal functional imager (RFI).

Methods: Normal subjects and patients with various ocular diseases underwent retinal blood flow imaging using the retinal functional imager.

Results: Twenty-eight eyes of 21 patients constituted the study population (eight eyes of six normal subjects and 20 eyes of 15 patients with different ocular diseases). Four patterns of retinal collateral circulation have been recognised: (1) looped collateral pattern - this arterovenous anastomotic vessel is characterised by a link between the endings of the adjacent artery and vein, and has an appearance of a loop; (2) vertical collateral pattern - this anastomotic vessel is characterised by a connection between superior and inferior vascular systems (arterial or venous), and in its course this collateral vessel crosses the horizontal raphe; (3) Hshaped collateral pattern - this arterovenous anastomotic vessel is characterised by a connection between two adjacent vessels (artery and vein), but, unlike anastomotic vessel of the "looped" pattern, this vessel connects the middle parts of the vessels and not the endings of these vessels; (4) cilioretinal-retinal collateral pattern. This anastomotic vessel is characterised by a link between the cilioretinal artery and retinal arterial circulation.

Conclusions: The findings of this study shed a different light on the retinal circulation and have demonstrated new patterns of retinal collateral circulation evident from RFI imaging. These patterns appear to be present in normal subjects as well as in patients with various ocular diseases.

A variety of invasive and non-invasive techniques have been employed in an attempt to fully appreciate the details of retinal blood flow and the associated vascular patterns. ${ }^{1-12}$ Major arteries and veins share a common territorial distribution. The larger arteries subdivide by dichotomous branching, while the smaller terminal arterioles tend to come off at right angels from the parent stem. The venous system has a similar arrangement. The terminal or precapillary arterioles, and the postcapillary venules form a regularly staggered arrangement and are linked by the interposed capillary bed.

There are no arteriovenous shunts in the normal retina, but some arteriovenous communications of the retina have been described and classified. Archer et al proposed a classification for arteriovenous communications of the retina. These included: grade I, retinal AV communication with interposition of an abnormal capillary plexus between major vessels; grade II, single or multiple direct AV communication without capillary bed; and grade III, extensive and complex AV communication without capillary bed, generally with visual loss and central nervous system lesions. ${ }^{13}$ Mansour et al proposed their classification: Grade I, an anastomosis between a small arteriole and a venule; Grade II, an anastomosis between a branch artery and a branch vein, and Grade III, a diffuse marked dilation of the whole vascular system. ${ }^{14}$ These communications are usually considered to be non-progressive.

With the introduction of the retinal functional imager (RFI), a new quantitative imaging system for studying retinal perfusion, it is now possible not only to estimate the retinal blood flow but also to visualise the microvasculature of the retina. ${ }^{15} \mathrm{~A}$ blood vessel changes its reflectance several times a second as erythrocytes pass in and out of it. This flickering allows detection of very small blood vessels from the motion within them. A vessel's "motion contrast" often exceeds its static contrast in a red-free fundus image. ${ }^{15}$

Using the RFI, we studied normal subjects and patients with different ocular diseases. Different patterns of retinal collateral circulation were identified and these observations are described in the present article.

\section{METHODS}

Normal subjects and patients with a variety of ocular diseases underwent retinal blood-flow imaging with the RFI (Optical Imaging, Rehovot, Israel) at the Retina Center of The New York Eye \& Ear Infirmary, New York, USA. The protocol was approved by the institutional review board at The New York Eye \& Ear Infirmary. All patients gave written informed consent prior to being examined with the RFI device.

\section{Retinal functional imager}

The RFI system (Optical Imaging, Rehovot, Israel) consists of a standard fundus camera, to which a customised stroboscopic flash lamp system and a fast digital camera have been added. ${ }^{15}$

The fine visualisation of retinal blood vessels, one of the main features of RFI, is made possible by the haemoglobin-filled erythrocytes in the bloodstream which provide a natural, high contrast (particularly at wavelengths between 530 and $590 \mathrm{~nm}$ ) chromophore which directly marks the flow of blood ${ }^{16}$ Tracking these erythrocytes gives a picture of the dynamic movement within the blood vessels. In other words, the RFI system detects the motion of red blood cells in small vessels using haemoglobin as an optical marker 


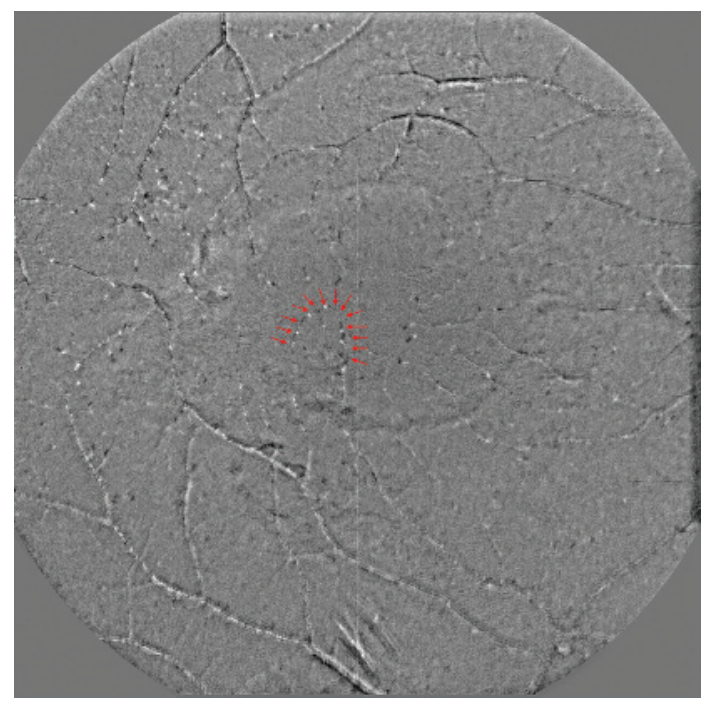

Figure 1 Retinal functional imager fundus images. Red arrows indicate "looped" collateral pattern.

instead of dye. A vessel's "motion contrast" often exceeds its static contrast in a red-free fundus image.

The patient is seated in front of the fundus camera, and a $60 \mathrm{~Hz}, 1024 \times 1024$ pixel digital imaging system takes snapshots of the fundus ( $35^{\circ}$ of the central retina) at rates high enough to reduce interframe retinal motion and follow erythrocytes moving at up to $20 \mathrm{~mm} / \mathrm{s}$. Eight consecutive flashes with an interflash interval of less than $20 \mathrm{~ms}$ are delivered to a patient. Sequences of eight frames are obtained from each patient as in standard fundus cameras. The captured eight frame sequences can be presented as a movie. These movies provide enhanced visualisation of retinal microvasculature. The RFI system has the capability of showing the direction of retinal blood flow within the blood vessel, making it possible to distinguish between artery and vein. Movies from each patient have been scrutinised for the presence of possible retinal collateral circulation. Different types of retinal collateral circulation were observed and described.

The circulatory patterns were correlated with fundus photography or fluorescein angiogram.

\section{RESULTS}

Twenty eight eyes of 21 patients constituted the study population (eight eyes of six normal subjects and 20 eyes of 15 patients with different ocular diseases). There were 11 men and 10 women, aged 23 to 85 years (mean 49 (SD 13) years).

The characteristics of the patients with ocular diseases were as follows: one eye had exudative age-related macular degeneration (AMD), three eyes had a dry AMD, one eye had non-proliferative diabetic retinopathy, six eyes had proliferative diabetic retinopathy, one eye had branch retinal vein occlusion, one eye had central serous retinopathy, one eye had sickle cell retinopathy, three eyes had posterior uveitis, one eye had choroidal nevus, one eye had glaucoma, and one eye had epiretinal membrane.

Four patterns of retinal collateral circulation have been recognised:

- "Looped" collateral pattern. This arterovenous anastomotic vessel is characterised by a link between the endings of the adjacent artery and vein, and has an appearance of a loop (fig 1).

- Vertical collateral pattern. This anastomotic vessel is characterised by a connection between superior and inferior

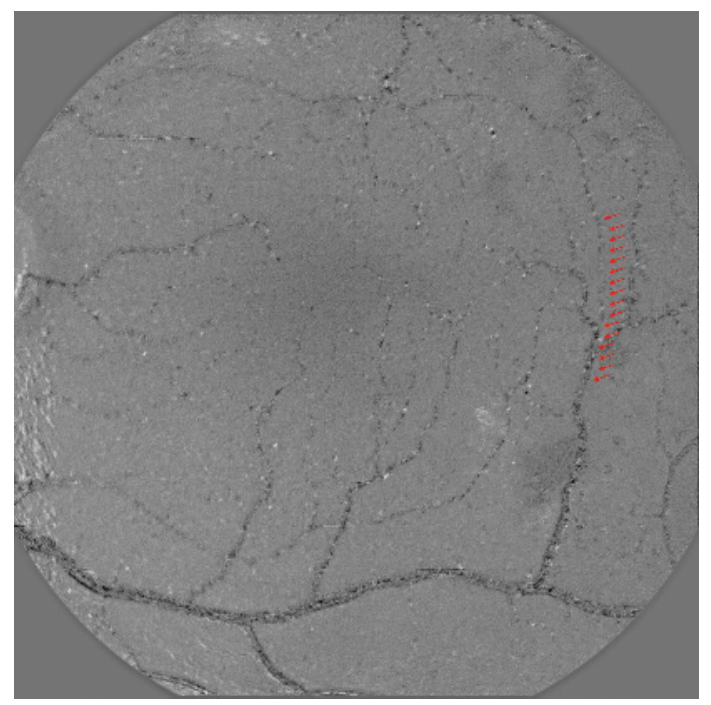

Figure 2 Retinal functional imager fundus images. Red arrows indicate vertical collateral pattern.

vascular systems (arterial or venous), and in its course, this collateral vessel crosses the horizontal raphe (fig 2).

- "H-shaped" collateral pattern. This arterovenous anastomotic vessel is characterised by a connection between two adjacent vessels (artery and vein), but, unlike anastomotic vessel of the "looped" pattern, this vessel connects the middle parts of the vessels and not the endings of these vessels (fig 3).

- Cilioretinal-retinal collateral pattern. This anastomotic vessel is characterised by a link between the cilioretinal artery and retinal arterial circulation (figs 4, 5).

The "looped" pattern was observed most frequently. Different types of the observed collateral patterns can coexist in the same patient. More detailed data are provided in table 1.

\section{DISCUSSION}

The findings of this study provide new information on retinal circulation and describe new patterns of collateral circulation, which may be present in normal subjects and patients with

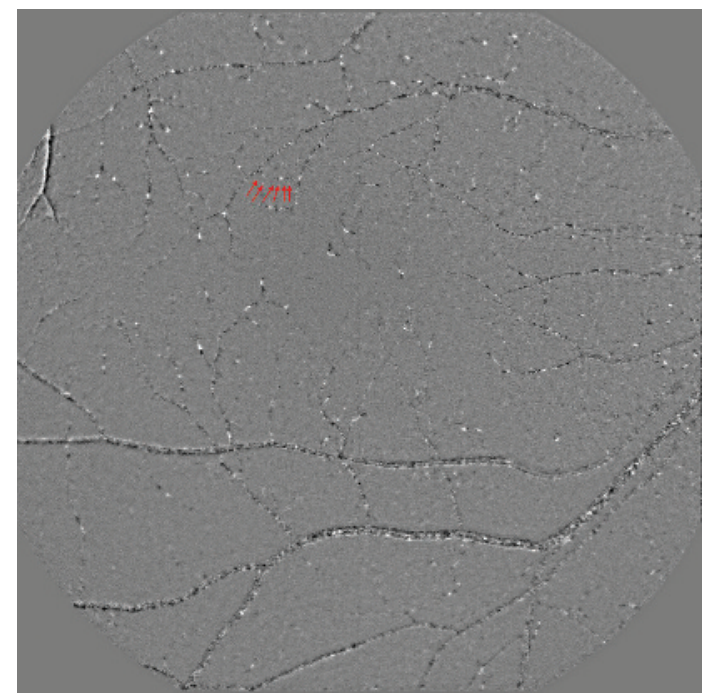

Figure 3 Retinal functional imager fundus images. Red arrows indicate "H-shaped" collateral pattern. 


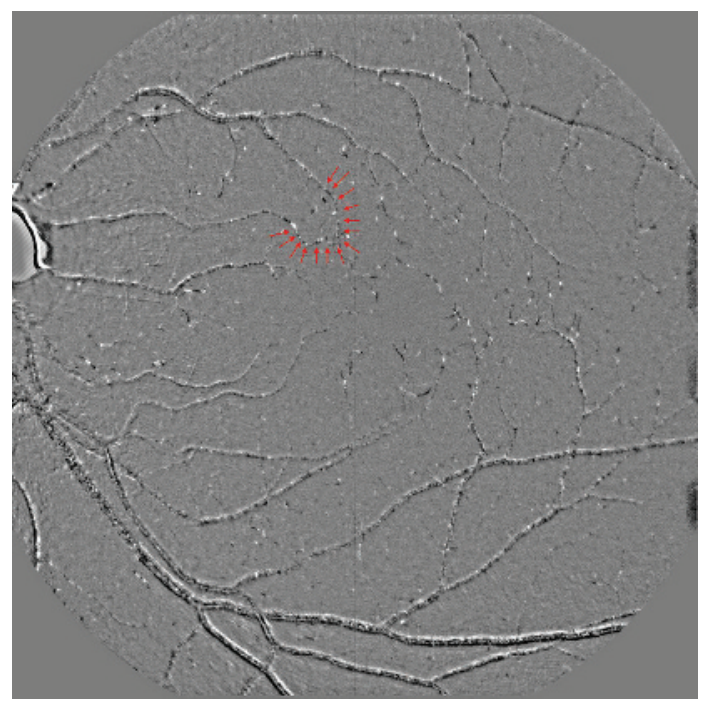

Figure 4 Retinal functional imager fundus images. Red arrows indicate cilioretinal-retinal collateral pattern.

various ocular diseases. These observations were made possible by the development of the RFI, a novel device for assessment of retinal blood flow and fine retinal vasculature. New patterns of retinal collateral circulation were found by the RFI.

There is uncertainty in the literature concerning the entities of retinal collaterals or retinal vascular shunts. Two decades ago, Henkind and Wise described retinal collaterals as vessels which develop within the framework of the existing retinal network and usually originating from the retinal capillary bed, joining obstructed to non-obstructed adjacent vessels or bypassing obstruction in a single vessel. Three subtypes of collaterals have been observed. The first is an arterioarteriolar subtype, which develops after branch artery obstruction. The second is a venovenular subtype, which develops after venous obstruction, and the third is an arteriovenous subtype, which occurs in situations of capillary bed obstruction, such as diabetic retinopathy, longstanding glaucoma and sickle cell retinopathy. Flow through these channels is generally slow and rarely normal. ${ }^{17}$

Collateral vessels can play a significant role in supplying oxygen to an organ, particularly when oxygen delivery is limited by disease in the normal vasculature. Collateral vessels can be pre-existing vessels that normally have little or no blood flow. Collateral blood vessels are particularly important in many organs, such as the coronary and skeletal muscle circulations. In the heart, collateral vessels can help to supply blood flow to ischaemic regions due to stenosis or occlusion of epicardial arteries. In the eyes, the development of collaterals is also very important and provides blood flow to ischaemic areas of the retina in cases of vascular occlusion events. The role of retinochoroidal collateral veins, also known as retinociliary or optociliary shunts/collaterals/veins or opticociliary anastomoses, which develop after a blockage of retinal venous drainage, has been extensively discussed in literature. Some studies have suggested that development of collaterals after central retinal vein occlusion (CRVO) is associated with an improved visual prognosis, ${ }^{18-20}$ whereas others have contradicted this statement. ${ }^{21-22}$ Collateral vessels that develop after branch retinal vein occlusion (BRVO) are different from those occurring after CRVO. Collateral channels may form and pass from the territory of the occlusion to a point proximal to the site of occlusion or to an uninvolved vein. The most characteristic type of collaterals are small, tortuous venous channels that cross the horizontal raphe (usually temporal to the fovea) and drain into the venous circulation of the uninvolved quadrant. Studies of (BRVO) patients have shown that collaterals had a favourable effect on visual prognosis. ${ }^{23}$

In contrast to retinal collaterals, the retinal vascular shunts are always arteriovenous communications, invariably dilated and tortuous, occupy almost the full retinal thickness, have a high flow rate and may be congenital (racemose angioma) or
Figure 5 Comparison photos of $(A)$ the retinal functional imager $(\mathrm{RFI}),(\mathrm{B})$ colour fundus and $(C, D)$ fluorescein angiography of one representative patient with a "looped" collateral pattern. The presence of this specific collateral pattern (red arrows) was obviously demonstrated by the RFI but was not clearly appreciated on colour and fluorescein angiogram fundus photos. Arterial (a) and venous (v) retinal vessels which are connected by this collateral branch are also designated.
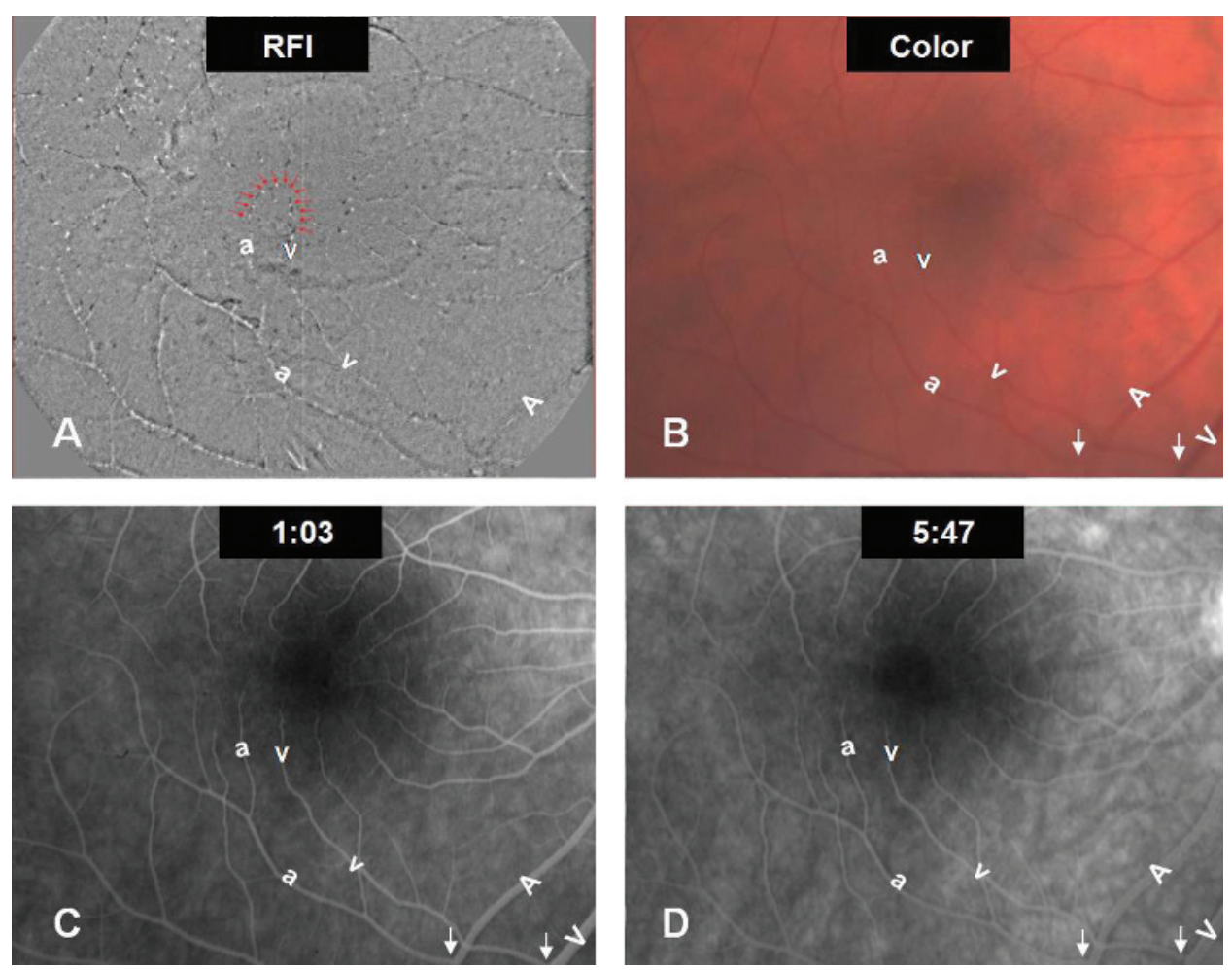
Table 1 Summarised data regarding presence (Y) or absence (N) of each collateral pattern in the study population

\begin{tabular}{|c|c|c|c|c|c|c|c|}
\hline \multirow[b]{2}{*}{ Eye (n) } & \multirow[b]{2}{*}{ Diagnosis } & \multirow[b]{2}{*}{$\begin{array}{l}\text { Laterality } \\
\text { (R/L) }\end{array}$} & \multirow[b]{2}{*}{$\begin{array}{l}\text { Gender } \\
\text { (M/F) }\end{array}$} & \multicolumn{4}{|c|}{ Collateral pattern } \\
\hline & & & & $\begin{array}{l}\text { Looped } \\
\text { (Y/N) }\end{array}$ & $\begin{array}{l}\text { Vertical } \\
\text { (Y/N) }\end{array}$ & $\begin{array}{l}\text { H-shaped } \\
\text { (Y/N) }\end{array}$ & $\begin{array}{l}\text { Cilioretinal-retinal } \\
\text { (Y/N) }\end{array}$ \\
\hline 1 & Dry age-related macular degeneration & $\mathrm{R}$ & M & Y & Y & $\mathrm{N}$ & $\mathrm{N}$ \\
\hline 3 & Choroidal nevus & L & $\mathrm{F}$ & Y & $\mathrm{N}$ & $\mathrm{N}$ & N \\
\hline 4 & Dry age-related macular degeneration & L & $\mathrm{F}$ & Y & $\mathrm{N}$ & $\mathrm{N}$ & $\mathrm{N}$ \\
\hline 5 & Central serous retinopathy & L & M & Y & Y & $\mathrm{N}$ & Y \\
\hline 8 & Glaucoma & R & $\mathrm{F}$ & Y & $\mathrm{N}$ & $\mathrm{Y}$ & $\mathrm{N}$ \\
\hline 9 & Epiretinal membrane & $\mathrm{R}$ & M & Y & $\mathrm{N}$ & $\mathrm{N}$ & Y \\
\hline 10 & Wet age-related macular degeneration & $\mathrm{R}$ & M & Y & $\mathrm{N}$ & Y & Y \\
\hline 11 & Dry age-related macular degeneration & L & $\mathrm{F}$ & $\mathrm{N}$ & $\mathrm{N}$ & $\mathrm{N}$ & Y \\
\hline 12 & Proliferative diabetic retinopathy & $\mathrm{R}$ & $\mathrm{F}$ & $\mathrm{N}$ & $\mathrm{N}$ & $\mathrm{Y}$ & $\mathrm{N}$ \\
\hline 17 & Proliferative diabetic retinopathy & L & $\mathrm{F}$ & $\mathrm{N}$ & Y & $\mathrm{N}$ & $\mathrm{N}$ \\
\hline 18 & Branch retinal vein occlusion & L & $\mathrm{F}$ & $\mathrm{N}$ & Y & $\mathrm{N}$ & N \\
\hline 19 & Proliferative diabetic retinopathy & $\mathrm{R}$ & M & $\mathrm{Y}$ & $\mathrm{N}$ & $\mathrm{N}$ & N \\
\hline 20 & Uveitis & $\mathrm{R}$ & $\mathrm{F}$ & $\mathrm{N}$ & $\mathrm{N}$ & $\mathrm{Y}$ & Y \\
\hline 21 & Normal & L & $\mathrm{F}$ & $\mathrm{N}$ & Y & Y & N \\
\hline 22 & Normal & $\mathrm{R}$ & M & Y & Y & $\mathrm{N}$ & $\mathrm{N}$ \\
\hline 23 & Normal & $\mathrm{R}$ & $\mathrm{F}$ & $\mathrm{N}$ & Y & $\mathrm{N}$ & Y \\
\hline 24 & Normal & L & $\mathrm{F}$ & $Y$ & $\mathrm{~N}$ & $\mathrm{~N}$ & $\mathrm{~N}$ \\
\hline 25 & Normal & L & $M$ & Y & $Y$ & $Y$ & $\mathrm{~N}$ \\
\hline 26 & Normal & $\mathrm{R}$ & $\mathrm{F}$ & $Y$ & $\mathrm{~N}$ & $\mathrm{~N}$ & $\mathrm{~N}$ \\
\hline 27 & Normal & $\mathrm{R}$ & $\mathrm{F}$ & $\mathrm{N}$ & Y & $\mathrm{N}$ & Y \\
\hline 28 & Normal & L & $\mathrm{F}$ & $Y$ & $\mathrm{~N}$ & $\mathrm{~N}$ & $\mathrm{~N}$ \\
\hline
\end{tabular}

F, female; L, left; $M$, male; $R$, right.

developmental (retinal angiomatosis, Coat disease). In these shunts, blood passes directly from the artery to the vein without going through the normal capillary bed. Flow in these vessels is usually rapid but may be slow if the shunt is fed by a vessel with a reduced flow.

Histopathology reveals dissimilarities between collateral and shunts: collaterals are the vessels with the same characteristics as the channel they link and occupy inner retinal layers, whereas shunts are larger thin-walled vessels, which may occupy the entire retinal thickness and even protrude from the retinal surface. ${ }^{17}$

The findings of this study suggest that there may be direct connections between arterial or venous circulation systems without involvement of the capillary bed. These connecting vessels clinically look like the vessels they connect and may lack tortuousity or dilation. They may be easily overlooked on the fluorescein angiogram and can be only detected using the RFI which shows the constant blood flow within retinal vessels.

These collaterals can be observed both in normal patients with no ocular disease and in patients with various ocular diseases. It is known that in some ocular vascular diseases, such as diabetic retinopathy, there is a capillary occlusion in the perifoveal network of patients with diabetes, and it is well described in histopathological studies. ${ }^{24}$ When this kind of occlusion occurs, the remaining capillaries take on the burden of maintaining normal blood flow and become thicker and look like the arterioles or venules they connect. Interestingly, none of six eyes with diabetic retinopathy which were included in the study showed evidence of cilioretinal-retinal collateral pattern.
These collateral vessels may have a wide range of appearances: on the one hand, they may look like normal small blood vessels connecting adjacent arterioles and venules; on the other hand, they may look like small and tortuous venous channels. The possibility exists that the part of the retinal collateral vessels, which are described in the present study, may represent the visualisation of small normal blood vessels or may imply the demonstration of the normal anatomy of retinal blood flow circulation.

RFI has a number of limitations. It is not a real-time device and must be synchronised with the heartbeats. In order to be able to analyse fine details of retinal vascularisation using the RFI, relatively clear ocular media are required. Eight frame images which are presented as a movie need to have a good resolution, so the detection of retinal collaterals in patients with opaque ocular media might fail. Instrumental artefacts may arise from imprecise focus and localisation during image acquisition. Another limitation of the RFI is the limited extent of the retina which could be analysed. RFI is capable of analysing $35^{\circ}$ of the central retina, so the peripheral retinal vasculature located beyond $35^{\circ}$ of the central retinal is out of range and could not be analysed. In addition, RFI shows only the functional retinal vasculature and does not show the capillaries, venules or arterioles that are no longer patent. However, the RFI has a unique ability to show circulatory pathways that cannot be seen by other imaging modalities. The development of newer versions of the RFI combined with conventional fluorescein angiography is currently in progress. These new combined imaging systems will be capable of 
demonstrating both functional and non-functional aspects of the retinal vasculature. In order to determine if the observed patterns can be attributed to a certain ocular disease and to determine the significance of the observed patterns of circulation, a study of a larger patient population is warranted in the future.

Acknowledgements: We thank H Savage and W Seiple for their helpful suggestions in preparing the manuscript.

Competing interests: None.

Ethics approval: Ethics approval was provided by The New York Eye and Ear Infirmary.

Patient consent: Obtained.

Provenance and peer review: Not commissioned; externally peer reviewed.

\section{REFERENCES}

1. Riva CE, Grunwald JE, Sinclair SH, et al. Blood velocity and volumetric flow rate in human retinal vessels. Invest Ophthalmol Vis Sci 1985;26:1124-32.

2. Riva CE, Petrig B. Blue field entoptic phenomenon and blood velocity in the retinal capillaries. J Opt Soc Am 1980;70:1234-8.

3. Bill A. Calorimetric procedures for the study of the blood flow through the ciliary region and the choroid in rabbits. Acta Ophthalmol 1962;40:131-48.

4. Bill A. Intraocular pressure and blood flow through the uvea. Arch Ophthalmol 1962;67:336-48.

5. Friedman E, Kopald HH, Smith TR. Retinal and choroidal blood flow determined with krypton-85 in anesthetized animals. Invest Ophthalmol Vis Sci 1964:3:539-47.

6. AIm A, Bill A. The oxygen supply to the retina. II: Effects of high intraocular pressure and of increased arterial carbon dioxide on uveal and retinal blood flow in cats. A study with radioactively labelled microspheres including flow determinations in brain and some other tissues. Acta Physiol Scand 1972;84:306-19.

7. Alm A, Bill A. Ocular and optic nerve blood flow at normal and increased intraocular pressure in monkeys (Macaca irus). A study with radioactively labelled microspheres including flow determination in brain and some other tissues. Exp Eye Res 1973:15:15-29

8. Yu DY, Alder VA, Cringle SJ, et al. Choroidal blood flow measured in the dog eye in vivo and in vitro by local hydrogen clearance polarography: Validation of a technique and response to raised intraocular pressure. Exp Eye Res 1988;46:289-303.

9. Gherezghiher T, Okubo H, Koss MC. Choroidal and ciliary body blood flow analysis: Application of laser Doppler flowmetry in experimental animals. Exp Eye Res 1991;53:151-6.

10. Riva CE, Cranstoun SD, Mann RM, et al. Local choroidal blood flow in the cat by laser Doppler flowmetry. Invest Ophthalmol Vis Sci 1994:35:608-18.

11. Klein GJ, Baumgartner RH, Flower RW. An image processing approach to characterizing choroidal blood flow. Invest Ophthalmol Vis Sci 1990;31:629-37.

12. Flower RW. Extraction of choriocapillaris hemodynamic data from ICG fluorescence angiograms. Invest Ophthalmol Vis Sci 1993;34:2720-9.

13. Archer DB, Deutman A, Ernest JT, et al. Arteriovenous communications of the retina. Am J Ophthalmol 1973;75:224-41.

14. Mansour AM, Walsh JB, Henkind P. Arteriovenous anastomosis of the retina. Ophthalmology 1987:94:35-40.

15. Nelson DA, Krupsky S, Pollack A, et al. Special report: Noninvasive multi-parameter functional optical imaging of the eye. Ophthalmic Surg Lasers Imaging 2005:36:57-66.

16. Jensen PS, Glucksberg MR. Regional variation in capillary hemodynamics in the cat retina. Invest Ophthalmol Vis Sci 1998;39:407-14.

17. Henkind $\mathbf{P}$, Wise GN. Retinal neovascularization, collaterals, and vascular shunts. Br J Ophthalmol 1974;58:413-22.

18. Priluck IA, Robertson DM, Hollenhorst RW. Long-term follow-up of occlusion of the central retinal vein in young adults. Am J Ophthalmol 1980;90:190-202.

19. Blinder KJ, Khan JA, Giangiacomo J, et al. Optociliary veins and visual prognosis after central retinal vein occlusion. Ann Ophthalmol 1989;21:192-7.

20. Fuller JJ, Mason J0, White MF, et al. Retinochoroidal collateral veins protect against anterior segment neovascularization. After central retinal vein occlusion. Arch Ophthalmol 2003;121:332-6.

21. Quinlan PM, Elman MJ, Kaur Bhatt $A$, et al. The natural course of central retinal vein occlusion. Am J Ophthalmol 1990;110:118-23.

22. Guiffre G, Palumbo C, Randazzo-Papa G. Optociliary veins and central retinal vein occlusion. Br J Ophthalmol 1993;77:774-7.

23. Im CY, Lee SY, Kwon OW. Collateral vessels in branch retinal vein occlusion. Korean J Ophthalmol 2002:16:82-7.

24. Yanoff M. Ocular pathology of diabetes. Am J Ophthalmol 1969;67:21-38. 